Hurley, MV, Semple, A, Sibley, F and Walker, A (2019) Evaluation of a health trainer-led service for people with knee, hip and back pain. Perspectives in Public Health, 139(6), pp. 308-315. Copyright () 2019 ( Royal Society for Public Health). DOI: https://doi.org/10.1177\%2F1757913919833721. 


\title{
Evaluation of a health trainer-led service for people with knee, hip and back pain
}

${ }^{1,2}$ Michael V Hurley, ${ }^{1}$ Amy Semple, ${ }^{1}$ Fay Sibley, ${ }^{1,2}$ Andrew Walker.

\author{
Affiliations: \\ ${ }^{1}$ Musculoskeletal Programme, Health Innovation Network. \\ ${ }^{2}$ Faculty of Health, Social Care and Education, St George's University of London and Kingston \\ University.
}

Corresponding author:

Professor Mike Hurley,

Faculty of Health, Social Care and Education,

St George's University of London and Kingston University,

6th Floor, Hunter Wing,

Cranmer Terrace,

London.

SW17 ORE

Email: michael.hurley@sgul.kingston.ac.uk

Key words

Hip pain, knee pain, back pain, osteoarthritis, health trainer, joint pain advisor 


\begin{abstract}
Introduction. Chronic knee, hip and back pain is extremely prevalent. Management guidelines emphasise maintaining physical activity and healthy weight to reduce pain, improve physical and mental wellbeing. Unfortunately, few people receive support to make lifestyle changes. We evaluated whether a health trainer-led, "joint pain advice" (JPA) service delivering person-centred lifestyle coaching was feasible, acceptable and effective for people with knee, hip and back pain.

Methods. Feasibility of delivering JPA service was assessed by documenting whether the health trainers could deliver JPA and its uptake. Nine health trainers delivered JPA. Participants were offered up to four appointments. At each appointment health trainers gave people information about their condition, co-developed care plans, suggested self-management strategies and used behavioural change techniques (motivational interviewing, goal-setting, action planning) to increase physical activity and reduce body weight. Pain, function, physical activity, and BMI were collected at baseline, 3-week, 6-week and 6-month. Focus groups captured people's opinions of the service's effectiveness, acceptability and usefulness.

Results. Of the 105 people who enquired about JPA 85 (81\%) used the service, after which their physical activity and function improved, pain and use of analgesia, BMI decreased. They felt more knowledgeable, and better motivated to adopt and maintain healthier behaviours. They attributed these improvements to the JPA service, because of better its consultations and collaborative holistic approach. Only a minority attended all 4 appointments because they felt they received sufficient advice from the initial appointments. The health trainers gained knowledge and skills to support clients with musculoskeletal conditions.
\end{abstract}

Conclusions. Using a holistic, patient-centred approach, health trainers can deliver lifestyle advice to people with chronic knee, hip or back pain safely, effectively and efficiently. The service was popular with recipients and health trainers, and helped people adopt healthier lifestyles that lead to reduced pain and other clinical improvements. 


\section{BACKGROUND}

Chronic joint pain, usually called osteoarthritis (OA), affects almost 9 million older adults the UK

${ }^{1,2}$. It adversely affects people's mobility, physical and psychosocial function, quality of life ${ }^{2,3}$ and increases the risk of obesity, diabetes, cardiovascular disease and depression, and it would be better to regard chronic joint pain as a public health problem ${ }^{4}$. OA health and social care expenditure is estimated to be approximately $1.5-2 \%$ of $\mathrm{GDP}^{5,6}$. As more people are living longer but adopting sedentary lifestyles and becoming more obese, the prevalence of OA and its personal and socioeconomic consequences will increase ${ }^{7,8}$.

Maintaining a healthy body weight and being physically active can reduce pain, improve function and quality of life ${ }^{9-11}$. However, people's poor understanding and erroneous health beliefs about the role of physical activity in the management of OA prevents them adopting healthy lifestyles ${ }^{12,13}$. OA is usually managed in primary care by GPs, but few GPs have the time or skills to enable people to adopt behaviours that improve symptoms (i.e. increase physical activity and lose excess weight) ${ }^{14-16}$.

Increased OA prevalence and demands on health services means there is pressure to deliver better care more efficiently ${ }^{17}$. Walker et al developed a Joint Pain Advisor (JPA) service, an allied health professional (AHP) led model of care that provides easier access to better care for people with knee and/or hip pain ${ }^{18}$. Delivered by physiotherapists in primary care practices, the JPA service used motivational interviewing and other behavioural change techniques to give people information and practical lifestyle advice emphasising the importance of weight control and physical activity ${ }^{11}$. The service reduced pain, weight, waist circumference, improved physical activity levels, function and quality of life. Additionally, participants were very happy with the service, and it reduced GP consultations and investigations.

Rather than a specific job or role JPA is better seen as service could be delivered by people other than healthcare professionals outside healthcare settings. If effective, this would improve access and efficiency even further. Health trainers are skilled health coaches using behavioural change techniques to encourage people to adopt healthier lifestyles ${ }^{19}, 20$ and incur lower workforce costs ${ }^{20}$. However, health trainers are not trained in the management of $\mathrm{OA}$, and are not confident about in giving self-management advice to people with joint pain or low back pain.

This study evaluated whether health trainers can deliver the JPA service in community settings. This is the first time "non-medically" trained professionals delivered advice to people with this ubiquitous problem in community settings, increasing access to better care for a large population of people that would benefit their physical and psychosocial well-being, and quality of life. 


\section{METHODS}

Design. A service evaluation of a community-based health trainer-led intervention in the Royal Borough of Greenwich, south London, using quantitative and qualitative methods to assess feasibility and effectiveness.

Participants. Participants were eligible to use the JPA service if they had clinical OA, that is they were $\geq 45$ years, had knee, hip or back pain for $\geq 3$ months, morning stiffness (if present) $<30$ min. Participants were identified and referred to the service from the Greenwich Live Well Service, primary care, local prevention programmes (such as 'Walking away from diabetes') or self-referral. Participants were excluded from using the service if they had acute musculoskeletal disorders, were unable to speak/understand English as they would be unable to understand the advice given.

Joint Pain Advisors. Ten health trainers were recruited to deliver the JPA service (hereafter called the Advisor). They had a minimum qualification of a City and Guilds Level 3 Health Trainer qualification, between 1-7 years experience delivering health coaching, some had motivational interviewing training. Advisors attended a one-day training course from the Health Innovation Network, Musculoskeletal team. Training covered basic joint anatomy, pathophysiology of OA, assessing and managing "red flag" symptoms, how healthy lifestyles can improve MSK health and myth busting about the 'dos and don'ts' about joint pain and physical activity and movement.

The JPA model has been described in more detail previously ${ }^{18}$, but briefly the consultations took place in 10 community sites across Greenwich. Participants were offered one 30-minute assessment and up to three review appointments, each about 30-45 minutes, at 3 weeks, 6 weeks and 6-month later (Table 1). The Advisors remit was to adopt a person-centred, holistic approach, building a collaborative relationship with each participant, using behaviour change techniques (e.g. motivational interviewing, goal setting, action planning, monitoring, positive feedback) to encourage and support the adoption of healthier lifestyles in line with NICE guidelines ${ }^{11}$.

\section{EVALUATION, DATA HANDLING AND ANALYSIS}

Feasibility. Referral rates, uptake, 'failure to attend' and adverse events were recorded to determine feasibility and acceptability of the service.

Clinical effectiveness. To evaluate the effectiveness of the JPA service delivered by health trainers quantitative clinical outcomes were collected at all appointments. The Hip or Knee Injury and Osteoarthritis Outcomes Score (HOOS/KOOS) was used as an OA-specific assessment of physical function, pain, and quality of life ${ }^{21}$. Physical activity was determined by the number of days per 
week participants reported they undertook $\geq 30$ minutes of moderate intensity physical activity. Waist circumference, BMI and the number of sit-to-stands a participants could perform from a chair without using their arms in 30 seconds were also recorded ${ }^{22}$. Statistical analysis was conducted in RStudio (Version 1.1.383, RStudio Inc.). Paired two sample t-tests was used to compare the means at baseline and review assessments.

People who cancelled or who did not return for a review appointment were contacted by telephone to ascertain why and to collect feedback on the service. This qualitative feedback gave an indication of the acceptability of the service.

Focus groups was conducted with 10 participants and 3 Advisors to determine people's experiences, opinions of, satisfaction with the JPA service, which shed light on the feasibility, acceptability and effectiveness of the JPA service when delivered by health trainers. For participants the aim was to understand what benefits they considered they had gained from attending the JPA service, for Advisors the aim was to explore their perceptions of delivering the service and understand how confident they felt when undertaking the role. A discussion schedule was used to guide the conversation. The focus groups were audio recorded and transcribed verbatim.

\section{RESULTS}

Participants. Participants were recruited through referral from health trainer's caseloads and a public promotional event to raise awareness of the JPA service. 105 participants enquired about the JPA service, of whom 85 (81\%) used the service. Participant's mean age was 67 (range 45 91 years), 76\% were female, 40 (47\%) had back pain, 33 (39\%) had knee pain, 12 (14\%) had hip pain, however, many reported a combination of knee and/or hip and/or back pain. Of these 69 (81\%) returned for the 3-week review, 45 (53\%) returned for 6-week review and $25(29 \%)$ returned for 6month review. The main reason for not attending review appointments was people had learned how to manage their pain better, had increased activity, felt better and did not need further help. Other reasons were ill health, conflicting engagements, the exercises were too difficult, did not wanted to see a health trainer.

Clinical outcomes. After seeing an Advsior improvements were evident in participant's physical function, i.e. number of sit to stands performed in 30 seconds increased by 6 , they walked at least 30 minutes more than 5 days per week ( 2 more days per week than at baseline), and their BMI and waist circumference dcreaeed by -0.22 and more than 5 centimetres respectively (Table 2). A large number of participants did not complete the $\mathrm{K} / \mathrm{HOOS}$ because they found them too lengthy or 
complicated, and there was insufficient data to determine changes in $\mathrm{K} / \mathrm{HOOS}$ at any of the assessments.

Participant focus group. Ten participants ( 9 women) attended the focus group with knee, hip and/or back pain. Four themes emerged from the discussions: (1) people's reasons for attending the JPA service, (2) their experience of the service, (3) the usefulness of the advice and support received, and (4) benefits obtained from the service (Table 3 ).

Reasons for attending. When asked their reasons for using the JPA service, the majority of people $(8 / 10)$ felt dissatisfied with the brief consultations and management they had received from their GP.

"...I was diagnosed with arthritis by the GP. He sat one side of the desk, me the other he went 'yeah that's arthritis' and scribbled a prescription - didn't even examine me. He gave me no advice on how to manage it - nothing"

"...Physio was a waste of time because going to the physio is exactly the same as what I've got from the pain clinic"

Some were existing service users of the health trainer service or healthy living classes, and had been identified through conversations with the health trainers who they trusted.

"...I was involved with the Health Trainer Service who said I should go to the Joint Pain

Advisor as it might benefit"

No one had been referred by their GPs, whom they presumed did not know about the service as it had not been mentioned to them during consultations.

Experience if the service. Participants were very positive about the JPA service. They liked the holistic, individualised approach, non-judgemental tone of the consultations, conducted in a nonclinical environments, which were not rushed allowing Advisors sufficient time to listen to them, explain the condition, how to manage it and to answer their questions.

"...I felt the Advisor had time to listen to me and me to them. It wasn't rushed - if it took 5 minutes to explain something that was fine..."

"... when you go to the GP you have this or that GP - tell them one thing and see another and they never know about you. This is good as you see the same person and they know you..." 
This fostered feelings of being cared for, and encouraged a collaborative relationship that made them want to make difficult lifestyle changes.

"...she didn't lecture me, worked with me, asking questions 'What do you think you can do?' and by the time I left I felt empowered to change..."

Advice and support. Prior to accessing the JPA service few people received information about their problem, the strong association between pain, excess weight and physical inactivity, and had not received any support that enabled them to modify poor beliefs and change their behaviour. After using the JPA service participants felt more knowledgeable about their condition, especially how exercise/activity could reduce pain,

"...knowing what $O A$ is and knowing the alternatives that are there to support you..."

"...I'm more able and willing to keep on the path with the knowledge l've been given..."

This helped break down entrenched fear-avoidance beliefs whereby people avoided being active for fear of causing themselves more pain and harm.

"...It is really important about the fear - it [service] took away the fear of doing exercise in case it makes it worse..."

"...before it was if my body is in pain it must be hurting - if it's hurting and I use it, it will make it worse not better. But when someone tells you 'you're not going to make it worse' you can change your mind-set. I now lead a walking group-would I have done that before JPA? No I wouldn't..."

They valued specific guidance about simple activities they could incorporate into their daily routines, e.g. walking and using stairs, and practical solutions such as pacing when exercising.

"...she helped me realise that exercise and moving it will help me maintain it [the knee].."

"...It enabled me to look at my diet - eating the same things but cooking them in less fat"

"...I've lost weight because, now I felt I wanted to..."

They appreciated the time spent by Advisors exploring and signposting to local and affordable activities they would enjoy.

"...she directed me to the local Tai Chi group and I'm a different person now..." 
Benefits. Participants reported a range of benefits such as reduced pain, reduced analgesia,

"...I was taking four or five painkillers a day - I don't take any now..."

“...not taking painkillers any more. I'm relying more on movement, exercise, better diet and eating habits..."

"...Before JPA my doctor gave me injections and tablets for my arthritis but now, because of the joint clinic I don't take as many - I take a couple in the morning and that's it..."

increased mobility, physical activity, independence with everyday activities e.g. getting dressed, gardening and walking, and adopting healthier diets".../ can get in and out the bath better now..."

"... can put my own tights on now..."

"...I can walk to my allotment faster - it used to take me 20 minutes now it is only 10..."

"...I am more active, walking more and doing yoga..."

They liked the more holistic approach compared to usual primary care

"...I found them even better than the GP because they took everything in - like nutrition they told me if I don't eat well I will have more pain - my diet was OK but I was cooking unhealthily..."

"...I think it is up to the GP to diagnose but when It is other issues - like I can't sit down or walk - all they can do is give you painkillers to ease the pain but the Advisor actually looks at the reasons why you know, it is happening. I think for most people, knowing what it is and knowing the alternatives that are there to support you, you can take it or leave it - and I think most people here have taken the advice..."

The informal, familiar, less clinical environment, which made them more receptive to the information being imparted to them

"...you are out of the clinic environment - feels less informal and therefore you are more open to what they are saying and sharing [information]..."

Advisor focus group. Three of the ten health trainers who delivered the JPA service attended a focus group. The findings were collated into five themes: (1) Understanding the intervention; (2) Enablers; (3) Barriers (4) Fit with existing services and (5) Competence, confidence and effects on practice (Table 4).

Understanding the intervention. The health trainers appreciated the need for the service and the issues within the healthcare system it sought to address. They emphasised the importance that 
needed to be placed on promoting non-pharmacological self-management strategies, in particular encouraging physical activity, and healthy eating

"...what we offer is a different understanding of how to manage pain through lifestyle changes..."

"...The same person would be going to the GP many times, prescribed many drugs but still no improvement and maybe side effects from the drugs - we are cheaper than GPs and save public money..."

and recognised the wider psychosocial benefits of working holistically with people to find solutions that work.

"....people were really desperate to find out what else would work [instead of the GP] and being able to support them..."

"...people were able to go out more, socialised more, being able to bath themselves without someone helping them. They were much happier in general to be honest..."

Enablers. The health trainers realised that having time to build rapport and relationships was integral to the success of the service, especially when helping people overcome their fears and concerns of exercise and activity.

"...we don't say you need to lose weight, give up smoking - the changes we help them come up with are the ones they are ready to make..."

“...people find out that pain doesn't damage - that really worked for my clients, it was the fear that held them back, not being confident to do exercise or scared to move as they thought they would damage their knee more. That was why this worked really well..."

They appreciated how knowledge of and signposting people to local activities and services increased the chance of people changing their behaviour.

“...knowing the services to refer to was a massive help. If you know they need more help you would be sitting there thinking I don't know what to do for this person - with depression or money problems. Whereas we move in those circles, we knew we could help people right here, right now..."

Barriers. They felt participants would have benefited from more frequent appointments and thought the time gap between appointments may have contributed to attrition.

"...it was definitely between the $3^{\text {rd }}$ and $4^{\text {th }}$ appointment (drop off) as it was a three month gap, which for some people is a long time..." 
Sometimes there was a mismatch between participants' expectations of what the service was offering, that in some cases affected the Advisor's ability to build collaborative relationships with participants. They felt raising GP's awareness of the JPA service would increase the number of referrals, as would linking it other community services such as social prescribing.

“..on the first session we had to make it clear what we did and didn't do-people accepted it but the initial letter needs to be clearer..."

"..some people wanted a scan but people who knew what we offered achieved it [managing the pain better]..."

Like many participants the health trainers felt the H/KOOS questionnaire too lengthy.

"...they are long compared with other projects l've worked on..."

"...It does take a long time - especially for those people with bad eyesight or can't read well"

Fit within existing services. Health trainers thought they were seen as an alternative to the traditional care offered and felt the service filled a gap in provision

".. a lot of them were on medication but it stops working as well as our systems get used to it, whereas the ice and heat, the Sit to Stands it really works and the outcomes are really goodit would really benefit to have it as a service..."

"...It would definitely work in the borough - or any borough - a lot of people say 'I go to the GP and they say your medication and see how it is. Whereas we can listen to them, try to help in a different way..."

and complemented existing services delivered locally where they could signpost people to, such as strength and balance classes, walking groups and cookery clubs.

"...the client really enjoyed the support we provided. She made so many changes in her lifestyle by joining exercise classes and cookery clubs in the local area. We've built the partnerships with the council, with other services..."

Competence, confidence and effects on practice. By the end of the study the health trainers felt acquisition of the new knowledge and skills about managing joint pain had enhanced their day-today practice.

"...we are using it every day..."

"..Once you have new knowledge you use it..." 
Where they had previously referred people with joint pain back to their GP, they now felt confident to support them in the community. They recognised they had positively impacted wider health determinants, such as mental wellbeing and social interactions. They felt the JPA service had become an integrated part of their role and they were going to continue to use the knowledge and skills they had developed.

"...before it would be "go back to your GP" but now we can help them...."

"... even with the Live Well service I can really use the joint pain advice and experience..." 


\section{DISCUSSION}

This study showed how simple training enables health trainers to deliver NICE advice to people with chronic joint pain facilitates access to better care, improves pain, physical, mental and social wellbeing and was highly valued by those delivering and receiving the service.

Current pathways of care for OA are unnecessarily complicated, costly, inefficient and delay or prevent most people receiving appropriate treatment and advice ${ }^{15}$. This has led to new ways of delivering high quality care ${ }^{17,23}$ whereby allied healthcare professionals successfully deliver care ${ }^{18,24,}$

${ }^{25}$ to people with uncomplicated musculoskeletal conditions avoiding unnecessary consultations, reducing waiting times, delays and costs currently associated with 'usual care' 18, 26, 27 .

Shortage of GPs, lack of training in behavioural change techniques and very brief consultations means that most people with uncomplicated joint pain receive suboptimal care ${ }^{28,29}$. People with OA are generally dissatisfied with their care, wanting to understand their condition better and be informed about effective treatments available ${ }^{30,31}$. The people who accessed our JPA service liked the ease of access, longer better quality consultations and it's person-centred approach ${ }^{18,32}$. This helped them achieve lifestyle changes with resulting improvements in their physical, mental and social wellbeing.

The JPA service is based on the biopsychosocial model of health where health is seen as being determined by a complex interaction of physical, emotional, mental and socioeconomic factors. This compels health care to address people's needs holistically. Moreover, for chronic conditions like OA, people need to understand why and how they can manage their problems by adopting lifestyles that minimise its affects and maximise their ability. To do this people need to understand the rationale behind self-management and be empowered to take control of their lives ${ }^{33}$. In addition, since the number of people with chronic joint pain is substantial and increasing rapidly, access to treatment by a large number of people needs to be greater than current healthcare systems can provide affordably. Health trainers work with people who have poor health beliefs and behaviours to adopt healthier lifestyles, such as smoking cessation, weight loss programmes, sexual health and increasing physical inactivity, often in hard to reach, intransigent populations ${ }^{19,34,35}$. Improving their knowledge, skills, and confidence about the important positive role physical activity and weight loss has in the management of joint pain, and their knowledge of local services that can be used to support good behaviour may be a more effective, efficient and sustainable way of helping the large numbers of people with chronic joint pain.

Strengths and limitations. This study was a pragmatic evaluation of a community-based service that recruited a representative population of people with joint pain and generated improvements 
very similar to a previous study ${ }^{18}$. These factors suggest the benefits of the service may be generalisable to wider populations of people with OA. However, several limitations need to be considered that might limit the inferences we can draw from this study. First, the number of people involved was small and a larger sample size is needed to substantiate the findings. Second, although qualitative and quantitative feedback from participants showed pain, function and life satisfaction had improved, people did not complete an outcome measuring pain, function and quality of life. This makes the findings difficult to compare our findings with other studies and interventions, and reaffirms the challenges associated with data collection in frontline services. Finally, the number of people returning for review declined. The reasons for non-attendance reflected our previous study where most chose not to attend because they had received sufficient reassurance and advice from the initial appointment ${ }^{18}$. We contend this does not reflect a failure of an ineffective or unneeded service, but rather participants choosing what help they need and when they needed it, they were doing exactly what we were encouraging them to do - self-managing their problem. This reinforces our argument that instead of creating a pathway that arbitrarily determines when people can access help, we need to create flexible, accessible, more efficient services that enable people to self-refer when they need it ${ }^{18,36,37}$.

In summary, a community-based service led by health trainers giving people with chronic joint pain better advice about self-management is deliverable, acceptable and improved clinical symptoms and outcomes. Rather than creating additional workforce roles, JPA is better seen as service that a range non-health professions could easily be trained to deliver in a range of nonhealthcare settings. Upskilling the existing workforce in this way offers a better, more accessible, more efficient, sustainable model of care that could fulfil the enormous unmet need of millions of people with chronic joint pain.

\section{Acknowledgements.}

This work was supported by an Health Innovation Network / Health Education England Innovation Grant 2018.

The authors would like to thank Sheila Taylor, the Royal Borough of Greenwich, Charlton Athletic Community Trust, the health trainers, the musculoskeletal programme team at the Health Innovation Network and all the participants who gave up their time and effort to take part in this study. 
Table 1. Joint Pain Advisor service model

\section{Timepoint (mode)}

Initial (baseline) consultation

(Face-to-Face)

3-week review

(Telephone)

6-week review

(Face-to-Face)

(Face-to-Face or telephone)

\section{Content of review appointment}

- Assessment of physical function, pain and symptoms, quality of life and lifestyle, Body mass index (BMI), waist circumference, number of Sit to Stands in 30 seconds and number of days physically active for 30 minutes or more a week.

- Co-development of an individualized care plan tailored to their needs based on NICE CG177: Management of OA:

- Increasing physical activity

- Simple pain management techniques (hot/cold packs; rest/activity cycling)

- Weight reduction, if necessary, to achieve and maintain a healthy body weight

- Signposting to activities in local area to support care plan e.g. exercise and healthy eating.

- Reinforcement of health messages and advice based on NICE CG177: Management of OA

- Provision of on-going support, reassurance, motivation and encouragement

- Performance against baseline measures (e.g. Sit to Stands, BMI, days physically active for 30 minutes or more a week)

- Participants baseline measures repeated and progress made fed-back

- Revision of goals (if appropriate)

- Reinforcement of health messages and advice based on NICE CG177: Management of OA

- Provision of on-going support, reassurance, motivation and encouragement

- Participants encouraged to take up activities through sign-posting if they haven't already done so

- As above and referred back to the GP if needed based to NICE CG177: Management of OA. 
Table 2. Clinical outcomes.

Review assessments

\begin{tabular}{|c|c|c|c|c|c|c|c|c|c|c|c|}
\hline \multirow{3}{*}{ Outcome } & & & \multicolumn{9}{|c|}{ Review assessments } \\
\hline & \multicolumn{2}{|c|}{ Baseline } & \multicolumn{3}{|c|}{ 3-weeks } & \multicolumn{3}{|c|}{ 6-weeks } & \multicolumn{3}{|c|}{ 6-months } \\
\hline & $\mathbf{n}$ & Mean (SD) & $\mathbf{n}$ & Mean (SD) & Change & $\mathrm{n}$ & Mean (SD) & Change & $\mathrm{n}$ & Mean (SD) & Change \\
\hline 30-second sit-to-stand /secs & 85 & $6.67(4)$ & 68 & $7.94(5)$ & 1.27 & 44 & $8.84(5)$ & $2.17^{*}$ & 25 & $12.64(7)$ & $5.97 * *$ \\
\hline Days walked 30 minutes per week / No. & 85 & $3.04(2)$ & 68 & $3.97(2)$ & $0.94 * *$ & 44 & $4.45(2)$ & $1.42^{* *}$ & 25 & $5.28(2)$ & $2.24 * *$ \\
\hline BMI & 85 & $31.98(7)$ & 27 & $32.09(6)$ & 0.11 & 26 & $31.39(6)$ & $-0.59 *$ & 17 & $31.76(7)$ & $-0.22 *$ \\
\hline Waist $(\mathrm{cm})$ & 85 & $103.32(18)$ & 20 & $106.10(16)$ & 2.78 & 20 & $100.6(16)$ & $-2.72 *$ & 12 & $98(2)$ & -5.32 \\
\hline
\end{tabular}

SD-Standard deviation

Secs-seconds

No. - number

BMI - Body Mass Index

Cn - cetrmetres

$*-p<0.05$

** $-p<0.01$ 


\section{References}

1. Arthritis Research UK. State of Musculoskeletal Health 2018: Arthritis \& other musculoskeletal conditions in numbers. Arthritis Research UK, 2018.

2. Conaghan PG, Porcheret M, Kingsbury SR, et al. Impact and therapy of osteoarthritis: the Arthritis Care OA Nation 2012 survey. Clinical Rheumatology. 2014: 1-8.

3. Hunter DJ, Schofield D and Callander E. The individual and socioeconomic impact of osteoarthritis. Nature Reviews Rheumatolology. 2014; 10: 437-41.

4. Arthritis Research UK. Musculoskeletal Health: a public health apporach. 2014.

5. Losina E, Walensky RP, Reichmann WM, et al. Impact of Obesity and Knee Osteoarthritis on Morbidity and Mortality in Older Americans. Annals of Internal Medicine. 2011; 154: 217-26.

6. Nuesch E, Dieppe P, Reichenbach S, Williams S, Iff S and Juni P. All cause and disease specific mortality in patients with knee or hip osteoarthritis: population based cohort study. BMJ. 2011; 342: d1165.

7. Anderson S and Loeser R. Why is osteoarthritis an age-related disease? Best Practice \& Research Clinical Rheumatology. 2010; 24: 15-26.

8. Muthuri SG, Hui M, Doherty M and Zhang W. What if we prevent obesity? Risk reduction in knee osteoarthritis estimated through a meta-analysis of observational studies. Arthritis Care \& Research. 2011; 63: 982-90

9. Hochberg MC, Altman RD, April KT, Benkhalti M, Guyatt G and McGowan J. American College of Rheumatology 2012 recommendations for the use of nonpharmacologic and pharmacologic therapies in osteoarthritis of the hand, hip, and knee. Arthritis Care Res (Hoboken). 2012; 64.

10. Fernandes L, Hagen KrB, Bijlsma JWJ, et al. EULAR recommendations for the non-pharmacological core management of hip and knee osteoarthritis. Annals of the Rheumatic Diseases. 2013.

11. National Institute for Health and Clinical Excellence. Osteoarthritis: the care and management of osteoarthritis in adults 2014

12. Hurley M. The role of muscle weakness in the pathogenesis of osteoarthritis. Rheumatic Diseases Clinics of North America. 1999; 25: 283-98.

13. Leeuw M, Goossens ME, Linton SJ, Crombez G, Boersma K and Vlaeyen JW. The fear-avoidance model of musculoskeletal pain: current state of scientific evidence. Journal of Behavioral Medicine. 2007; 30: 77-94.

14. Martin B and Adrian E. Assessing appropriateness of osteoarthritis care using quality indicators: a systematic review. Journal of Evaluation in Clinical Practice. 2015; 21: 782-9.

15. Foster N, Hartvigsen $\mathrm{J}$ and Croft $\mathrm{P}$. Taking responsibility for the early assessment and treatment of patients with musculoskeletal pain: a review and critical analysis. Arthritis Research \& Therapy. 2012; 14: 205.

16. Hagen KB, Smedslund G, Østerås N and Jamtvedt G. Quality of Community-Based Osteoarthritis Care: A Systematic Review and Meta-Analysis. Arthritis Care \& Research. 2016; 68: 1443-52.

17. Tavabie JA and Simms JM. Career planning for the non-clinical workforce - an opportunity to develop a sustainable workforce in primary care. Educ Prim Care. 2017; 28: 94-101.

18. Walker A, Williams R, Sibley F, Stamp D, Carter A and Hurley M. Improving access to better care for people with knee and/or hip pain: service evaluation of allied health professional-led primary care. Musculoskeletal Care. 2018; 16: 222-32.

19. Carr SM, Lhussier M, Forster N, et al. An evidence synthesis of qualitative and quantitative research on component intervention techniques, effectiveness, cost-effectiveness, equity and acceptability of different versions of health-related lifestyle advisor role in improving health. Health Technol Assess. 2011; 15: iii-iv, $1-284$. 
20. Visram S, Clarke C and White M. Making and Maintaining Lifestyle Changes with the Support of a Lay Health Advisor: Longitudinal Qualitative Study of Health Trainer Services in Northern England. PLOS ONE. 2014; 9: e94749.

21. Roos E and Lohmander LS. The Knee injury and Osteoarthritis Outcome Score (KOOS): from joint injury to osteoarthritis. Health and Quality of Life Outcomes. 2003; 1: 64.

22. Jones CJ, Rikli RE and Beam WC. A 30-s Chair-Stand Test as a Measure of Lower Body Strength in Community-Residing Older Adults. Research Quarterly for Exercise and Sport. 1999; 70: 113-9.

23. Health Education England. The future of primary care: Creating teams for tomorrow London:. Available at: https://www.hee.nhs.uk/sites/default/files/documents/WES_The-future-of-primary-care.pdf [Accessed 23 Sep. 2017]. London, 2015.

24. Ludvigsson ML and Enthoven P. Evaluation of physiotherapists as primary assessors of patients with musculoskeletal disorders seeking primary health care. Physiotherapy. 2012; 98: 131-7.

25. Hurley M, Walsh N, Bhavnani V, Britten N and Stevenson F. Health beliefs before and after participation on an exercised-based rehabilitation programme for chronic knee pain: Doing is believing. $B M C$ Musculoskeletal Disorders. 2010; 11: 31.

26. Holdsworth LK, Webster VS and McFadyen AK. What are the costs to NHS Scotland of self-referral to physiotherapy? Results of a national trial. Physiotherapy. 2007; 93: 3-11.

27. Nordeman L, Nilsson B, Möller M and Gunnarsson R. Early Access to Physical Therapy Treatment for Subacute Low Back Pain in Primary Health Care: A Prospective Randomized Clinical Trial. The Clinical Journal of Pain. 2006; 22: 505-11.

28. Denoeud L, Mazieres B, Payen-Champenois C and Ravaud P. First line treatment of knee osteoarthritis in outpatients in France: adherence to the EULAR 2000 recommendations and factors influencing adherence. Ann Rheum Dis. 2005; 64.

29. Ganz DA, Chang JT, Roth CP, et al. Quality of osteoarthritis care for community-dwelling older adults. Arthritis Rheum. 2006; 55: 241-7.

30. Mitchell HL and Hurley MV. Management of chronic knee pain: A survey of patient preferences and treatment received. BMC Musculoskeletal Disorders 2008; 9: 123.

31. Sanders C, Donovan J and Dieppe P. The significance and consequences of having painful and disabled joints in older age: co-existing accounts of normal and disrupted biographies. Sociology of Health \& Illness. 2002; 24: 227-53.

32. Ndosi M, Lewis M, Hale C, et al. The outcome and cost-effectiveness of nurse-led care in people with rheumatoid arthritis: a multicentre randomised controlled trial. Annals of the Rheumatic Diseases. 2013.

33. McCarley P. Patient empowerment and motivational interviewing: engaging patients to self-manage their own care. Nephrol Nurs J. 2009; 36: 409-13.

34. Vansteenkiste $\mathrm{M}$ and KM. S. There's nothing more practical than a good theory: Integrating motivational interviewing and self-determination theory. British Journal of Clinical Psychology. 2006; 45: 63-82.

35. Ekong $\mathrm{G}$ and Kavookjian J. Motivational interviewing and outcomes in adults with type 2 diabetes: A systematic review. Patient Education and Counseling. 2016; 99: 944-52.

36. Hewlett S, Kirwan J, Pollock J, et al. Patient initiated outpatient follow up in rheumatoid arthritis: six year randomised controlled trial. BMJ. 2005; 330: 171-5.

37. Maddison P, Jones J, Breslin A, Barton C, Fleur J and Lewis R. Improved access and targeting of musculoskeletal services in northwest Wales: targeted early access to musculoskeletal services (TEAMS) programme. BMJ. 2004; 329. 